\title{
Intra-arterial treatment in acute ischaemic stroke:What we learn from the MR CLEAN trial
}

\author{
A Sekhar \\ Consultant Neurologist, Walton Centre NHS Foundation Trust, Liverpool, UK
}

TITLE A Randomized Trial of Intraarterial Treatment for Acute Ischemic Stroke

AUTHORS Berkhemer OA, Fransen PS, Beumer D et al.

JOURNAL N Engl J Med 20I5; 372: II-20. http://dx.doi.org/l0.1056/ NEJMoa I 4 I I 587.

DECLARATION OF INTERESTS No conflicts of interests declared

\author{
Correspondence to A Sekhar \\ Walton Centre NHS Foundation \\ Trust \\ Liverpool L9 7LJ \\ UK
}

e-mail alakendu.sekhar@ thewaltoncentre.nhs.uk

\section{SUMMARY}

Intra-arterial treatment increases the likelihood of recanalisation in patients with acute ischaemic stroke caused by proximal intracranial occlusion. The purpose of the Multicenter Randomized CLinical trial of Endovascular treatment for acute ischemic stroke in the Netherlands (MR CLEAN) was to assess the safety and efficacy on functional outcome of intra-arterial treatment in these patients.

The trial was a pragmatic, phase III, multicentre randomised clinical trial with blinded outcome assessment. The trial aimed to address the treatment effect of intra-arterial intervention vs no further intervention in patients with acute ischaemic stroke with large vessel occlusion. All patients had received standard care $[89 \%$ had intravenous alteplase, tissue-type plasminogen activator, (IV tPA)].

The study population was 500 patients with ischaemic stroke with a score on the National Institute of Health stroke scale (NIHSS) of 2 points or more, relevant intracranial arterial occlusions demonstrated by neuroimaging, and the possibility of starting endovascular treatment within 6 hours of stroke onset.

The exact choice of endovascular treatment modality was left to the discretion of the local investigator and the treating physicians. Intra-arterial thrombolysis with either urokinase or alteplase, mechanical treatment, or both, were permitted. Mechanical treatment consisted of retraction or aspiration of the thrombus with a catheterguided device, or stenting. Most patients $(81.5 \%)$ in the treatment arm had the Solitaire stent retriever device.

The randomisation was stratified for use of intravenous alteplase, planned treatment modality (intraarterial thrombolysis, mechanical thromb-ectomy or both) and centre.
The primary outcome was the modified Rankin Score (mRS) 90 days after inclusion in the study. The mRS is a 7-point scale ranging from 0 (no symptoms) to 6 (death). A score of 2 or less indicates functional independence. The effect of treatment was estimated by means of ordinary logistic regression (shift analysis), which considered the whole range of the $\mathrm{mRS}$.

The secondary outcomes were the NIHSS score at 24 hours, vessel patency at 24 hours, infarct size at days 5-7, and occurrence of major bleeding.

Compared with usual therapy, the group assigned to intra-arterial treatment had significantly improved outcomes at 90 days as determined by lower scores on the $\mathrm{mRS}$ (adjusted common odds ratio $1.67,95 \% \mathrm{Cl}$ I.2I-2.30).

The intra-arterial treatment group had a significantly higher rate of functional independence (i.e. a mRS score of $0-2)$ at 90 days compared with usual treatment (32.6 vs $19.1 \%$, absolute risk difference, $13.5 \%$, 95\% Cl 5.9-2।.2).

Several other imaging and clinical measures also favoured intra-arterial treatment, including:

- Average reduction of 2.9 points in NIHSS score after $5-7$ days ( $95 \% \mathrm{Cl}$ I.5-4.3 points)

- Successful recanalisation at 24 hours: rate of $75.4 \%$ vs $32.9 \%$ (adjusted OR 6.88; 95\% Cl 4.34-10.94)

- Decrease in median infarct volume of $19 \mathrm{~mL}(95 \%$ Cl 3-34 mL)

\section{OPINION}

The MR CLEAN trial provides further evidence that mechanical treatment for the subset of stroke patients with large vessel occlusion is superior to conventional IV thrombolysis alone, and as safe. 
The current 'gold standard' treatment for acute ischaemic stroke is IV thrombolysis. A critical analysis of the data, however, raises some doubts about its efficacy.' Although IV t-PA may be effective, several factors, including the location, size, and characteristics of the thrombus, may lead to tPA resistance in about $50 \%$ of patients. ${ }^{2}$

We already know that the chances of recanalisation with IV t-PA are even more limited in cases of proximal large artery occlusions. In a landmark study ${ }^{3}$ it was shown that the overall chances of recanalisation with IV thrombolysis are around $30 \%$, but less when the thrombi are in the middle cerebral artery stem $(24 \%)$ or in the proximal internal carotid artery (8\%).

A meta-analysis by Rha and Saver $^{4}$ suggested a spontaneous recanalisation rate of $24 \%$ with increment to $46 \%$ after IV t-PA treatment.

MR CLEAN is a successful stent retrieval trial which proves the superiority of the Solitaire stent retrieving device over the standard stroke care (IV thrombolysis) in ischaemic strokes secondary to large vessel occlusions. Since its publication two further positive trials, ESCAPE ${ }^{5}$ and EXTEND-IA ${ }^{6}$, evaluating mechanical thrombectomy in acute ischaemic stroke have been published.

To deliver such sophisticated and precision stroke intervention requires correct patient identification and timely intervention by experienced neurointervention specialists. The key in identifying appropriate patients requires a $24 \mathrm{~h}$ computed tomography angiogram service in stroke centres. The diagnostic radiologist must identify the clot location and there must be rapid triaging to the stroke intervention centre. In the UK the provision of computed tomography angiograghy is patchy and there are very few centres that perform such complex neurovascular interventions on a regular basis. It is probably time to train more interventional neuroradiologists so that this service can be delivered in a more equitable fashion. For the time being, a regional stroke rota staffed by a pool of interventional radiologists may be an option. It may also be helpful to train some neurologists and stroke physicians as neurointerventionists (in an analogous fashion to the training some cardiologists receive).

We have to remember this intervention is only fruitful in correctly identified patients. It is, of course, not without risk of unfavourable outcomes, with the added complications of malignant cerebral oedema after reperfusion. The proportion of hemicraniectomies (6.0 vs $4.9 \%$ ) was higher in the intra-arterial treatment group.

It is perhaps best for this intervention to be provided in regional neurosciences centre with neurosurgical and neurointensive care support available. These patients should be treated as a priority. It is time for UK stroke physicians, neurologists, interventional neuroradiologists and ambulance services to join forces to formulate a plan so that we are not left so far behind our Dutch counterparts.

Finally it is important to note that the II\% of patients in MR CLEAN and $25 \%$ in ESCAPE who did not receive IV tPA did not get less benefit from thrombectomy. This raises the question of whether we should move to immediate endovascular therapy, bypassing IV thrombolysis as this uses up precious time in the immediate aftermath of an acute ischaemic stroke.

\section{REFERENCES}

I Wardlaw JM, Murray V, Berge E et al. Recombinant tissue plasminogen activator for acute ischaemic stroke: an updated systematic review and meta-analysis. Lancet 20I2; 379: 2364-72. http://dx.doi.org/I0.I0I6/S0I40-6736(I2)60738-7.

2 Balami JS, Hadley G, Sutherland BA et al. The exact science of stroke thrombolysis and the quiet art of patient selection. Brain 2013; 136: 3528-53. http://dx.doi.org/I0.1093/brain/awt20 I

3 del Zoppo GJ, Poeck K, Pessin MS et al. Recombinant tissue plasminogen activator in acute thrombotic stroke and embolic stroke. Ann Neurol 1992; 32: 78-86.

4 Rha JH, Saver JL. The impact of recanalization on ischemic stroke outcome: a meta-analysis. Stroke 2007; 38: 967-73.

5 Goyal M, Demchuk AM, Menon BK et al. Randomized assessment of rapid endovascular treatment of ischemic stroke. $N$ Engl J Med Epub ahead of print II Feb 20I5. http://dx.doi.org/I0.1056/ NEJMoa I4I4905.

6 Campbell BC, Mitchell PJ, Kleinig TJ et al. Endovascular therapy for ischemic stroke with perfusion-imaging selection. N Engl J Med Epub ahead of print II Feb 20I5. http://dx.doi.org/I0.1056/ NEJMoa I4|4792 